\title{
Noninvasive Monitoring of Vital Signs Based on Highly Sensitive Fiber Optic Mattress
}

\author{
Senmao Wang, Xiaoling Ni*, Liangye Li, Jingyi Wang, Qi Liu, Zhijun Yan, \\ Lin Zhang and Qizhen Sun.
}

\begin{abstract}
A smart mattress based on optical fiber Mach-zender interferometer (OF-MZI) is designed for noninvasive and continuous monitoring of human vital signs. Through arranging the sensing fiber between two elastic covering layers with sandwich structure, the mattress was sensitive to the respiration and heartbeat induced micro-pressure. In the processing terminal, the waveforms of vital signs were demodulated by $3 * 3$ coupler based differentiate and cross-multiplying method, and then four characteristic indicators including the heart rate, heartbeat amplitude, respiration rate, and respiration amplitude were respectively extracted through feature extraction algorithm, for evaluating the human health condition. Clinical experimental results of eighteen subjects indicate that the mattress system could not only distinguish the activity states of no body, on bed, body movement and off bed, but also contribute to clinical diagnosis of bradycardia, tachycardia, polypnea and apnoea. By adopting Bland-Altman analysis method, good reproducibility and accuracy were confirmed, where the max errors of heart rate and respiration rate are respectively $2 \mathrm{bpm}$ and $1 \mathrm{bpm}$. Moreover, the responses at different positions of the mattress are identical and the continuous monitoring results in one day are consistent with daily change of vital signs, which proves that the fiber optic mattress has good reliability and stability. Beneficial from high-sensitivity, multiple parameters, long-term continuous monitoring, high comfortability and low cost, the mattress is promising in the early detection and prevention of cardiac and respiratory diseases as a household medical device.
\end{abstract}

Index Terms-smart mattress, optical fiber Machzender interferometer, healthcare monitoring, heartbeat, respiration.

\section{INTRODUCTION}

$\Psi^{n}$ $\mathrm{n}$ recent years, the early detection and prevention of cardiac and respiratory diseases have attracted worldwide

This work was supported by the Wuhan Morning Light Plan of Youth Science and Technology (No. 2017050304010280), National Natural Science Foundation of China (NSFC) under Grant 61922033\&61775072, the Science Fund for Creative Research Groups of the Natural Science Foundation of Hubei under Grant 2018CFA004, and the Fundamental Research Funds for the Central Universities under Grant 2019kfyRCPY095. (Corresponding author: Xiaoling $\mathrm{Ni}$ )

S. Wang, L. Li, J. Wang, Q. Liu, Z. Yan and Q. Sun are with School of Optical and Electronic Information, National Engineering Laboratory for Next Generation Internet Access System, Huazhong University of Science attention. Heartbeat and respiration are two important indicators to reveal the human health condition, which are closely related to cardiac and respiratory diseases. By chronically measuring the rate and amplitude of heartbeat, people can effectively monitor the cardiac activities and then diagnose heart diseases. While, respiration monitoring can help people to understand the physiological and pathological states of respiratory tract, alveolar and thoracic movements, and further to provide valuable information for respiratory diseases diagnosis. Compared with invasive measurement, noninvasive monitoring of vital signs is more convenient and in capable of ensuring the measurement safety, which is significant in the modern medical monitoring fields.

Numerous noninvasive techniques have been developed to monitor the human vital signs, which can be classified as electrical and optical sensors. For electrical sensors, camera sensor [1], ultrasonic proximity sensor [2], and multi-walled carbon nanotubes sensor [3] were proposed to realize respiration measurement. In 2012, H. J. Baek et al. integrated capacitively coupled electrodes with a chair to record heartbeat waveforms [4]. Furthermore, the sensors based on polyvinylidene fluoride (PVDF) piezopolymer film [5], static charge-sensitive-bed (SCSB) [6], ECG [7], Doppler radar [8], acoustic sensor [9], air mattress [10] and liquid pressure mattress [11] have been adopted to simultaneous measure respiration rate and heart rate. However, electrical sensors suffer from electromagnetic interference, short life, and easy-corrosion, which is not suitable for long-term medical monitoring.

Optical fiber sensors intrinsically offer many advantages over traditional electrical sensor such as flexibility, electromagnetic interference immunity and high resistance to moisture, which have been widely applied in medical testing fields. Various optical fiber sensors have been developed for monitoring cardiac and respiratory activities. Among them, distributed Bragg reflector (DBR) fiber laser sensor [12] and fiber Bragg grating (FBG) sensor [13] have high sensitivity but require costly interrogation system. Further, the low-cost schemes have emerged, including Photoplethysmography (PPG) sensor [14], polymer optical fiber sensor [15] and

and Technology, Wuhan 430074, Hubei, P. R. China (e-mail: wsm@hust,edu.cn; m201972221@hust.edu.cn; jingyiwang@hust.edu.cn; oewonder@ hust.edu.cn; yanzhijun@gmail.com; qzsun@ mail.hust.edu.cn).

$\mathrm{X} . \mathrm{Ni}$ is with Hospital of Huazhong University of Science and Technology, Wuhan 430074, Hubei, P. R. China. (e-mail: nixiaoling@hust.edu.cn).

L. Zhang is with the Aston Institute of Photonic Technologies, Aston University, Birmingham, B4 7ET, U.K. (e-mail: 1.zhang@ aston.ac.uk). 
hetero-core fiber optic pressure sensor [16]. However, PPG sensor suffers from the poor stability and reliability at the condition of finger movement or variations in human skin tone. Polymer optical fiber sensor and hetero-core fiber optic pressure sensor have the drawback of complex fabrication process. Later, several simple sensors such as photonic crystal fiber sensors [17-18], and micro-bend optical fiber [19] were investigated. Whereas, photonic crystal fiber sensors and micro-bend optical fiber sensor may cause poor comfortability on account of skin contacting and hard sensitization structure, respectively.

In this paper, we proposed a fiber optic MZI based smart mattress system to monitor human vital signs, which is consisted of a sensing mattress and a processing terminal. Specifically, the sensing mattress is constructed by sandwiching single-mode fiber (SMF) with two polyvinyl chloride (PVC) layers, and the processing terminal carries phase demodulation method and feature extraction algorithm. Consequently, high-fidelity vital signs signals can be synchronously recovered and then four indicators of heart rate (HR), heart amplitude (HA), respiration rate (RR), and respiration amplitude (RA) can be calculated for early diagnosis and prevention of cardiac and respiratory diseases. In the clinic experiment, the activity states of no body, on bed, body movement and off bed were distinguished. In terms of the four indicators, the health condition of eighteen subjects and corresponding errors of HR and RR were analyzed. In addition, two reliability test experiments were also carried out. The fiber optic mattress has the advantages of low cost, multiple physiological parameters monitoring, user-friendly, simple fabrication and high comfortability, which is especially suitable for household medical and longterm monitoring.

\section{MATERIALS AND METHOD}

\section{A. Sensitization principle}

High sensitivity of the mattress system to heartbeat and respiration is realized by adopting phase modulation method and package sensitization. Optical fiber Mach-zender interferometer (OF-MZI) is a kind of sensors with high sensitivity and simple fabrication, which is capable of measuring strain, pressure, and vibration based on phase modulation. As depicted in Fig. 1, the OFMZI is consisted of a continuous wave distributed feed-back laser with coherent light, two couplers, an optical fiber reference arm, an optical fiber sensing arm, and three photodetectors (PD). The first

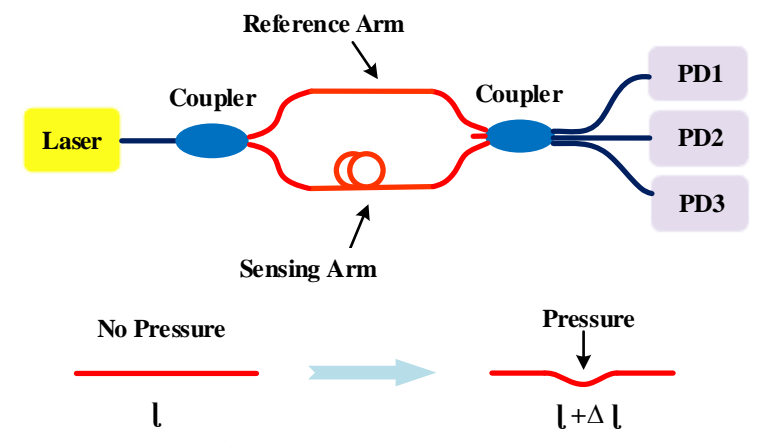

Fig. 1. The structure of OF-MZI
$3 \mathrm{~dB}$ coupler is used to split the laser power into two parts with equal light intensity, and then transmitted into the reference fiber and sensing fiber, respectively. Finally, the sensing light is interfered with the reference light through a symmetric $3 * 3$ coupler and then detected by the PDs. Owing to the photo-elastic effect of optical fiber [20], the optical phase of light in the sensing fiber will be modulated by the pressure applied on the sensing arm. As a result, the phase difference between the sensing light and reference light, $\Delta \varphi$, can be described as follow,

$$
\Delta \varphi=\beta\left[1-\frac{n}{2}\left(P_{l}+P_{t}\right)\right] \Delta l \propto \varepsilon_{z}
$$

Where $\beta$ is the propagation constant of the light, $\mathrm{n}$ is the refractive index of the fiber core, $P_{l}$ and $P_{t}$ are the photoelastic coefficients of single-mode silica fiber, $\Delta \mathrm{l}$ is the length change of fiber, $\varepsilon_{z}$ is the axial strain of the sensing fiber. $\beta, \mathrm{n}, P_{l}$, and $P_{t}$ are all the constants which are dependent on fiber properties, so the optical phase difference is proportional to the length change of fiber and is correspondingly proportional to the axial strain of fiber.

In order to recover the phase difference $\Delta \varphi$ from the detected light, the demodulation method based on a symmetric $3 * 3$ coupler is employed to OFMZI. Theoretically, there is phase shift of $120^{\circ}$ between every two adjacent output arms of the $3 * 3$ coupler [21]. Hence, the light power from the three output ports of the symmetric $3 * 3$ coupler can be given by

$$
\begin{gathered}
I_{1}=I_{0}[A+B \cos (\Delta \varphi-2 \pi / 3)] \\
I_{2}=I_{0}[A+B \cos (\Delta \varphi)] \\
I_{3}=I_{0}[A+B \cos (\Delta \varphi+2 \pi / 3)]
\end{gathered}
$$

Where $I_{o}$ is the intensity of the light induced by the distributed feedback laser, A and B are constants related to the input optical power. Through differentiate and crossmultiplying demodulation algorithm [22-24], the phase difference $\Delta \varphi$ can be extracted.

Owing that the silica fiber is insensitive to the pressure [25], polymer material is used to package the sensing fiber as a pressure transducer in this work. Fig. 2. shows the transverse section of the packaging structure, in which the fiber is tightly bonded with the packaging material. The packaging and fiber coating materials are respectively polyvinyl chloride (PVC) and acrylic resin, which can be regarded as linear elastic materials [26-28]. So, the axial elongation of polymer material induced by external pressure will generates the axial strain of the sensing fiber. That is to

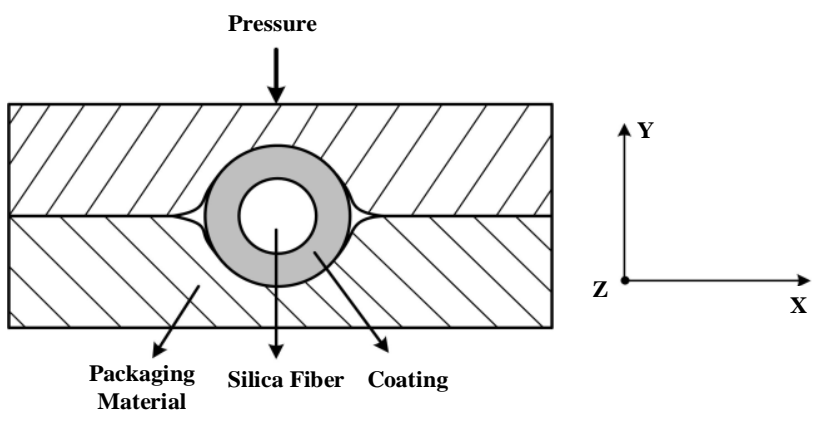

Fig. 2. The transverse section of packaging structure 
TABLE I

MATERIAL PARAMETERS

\begin{tabular}{llll}
\hline \hline Material & $\begin{array}{l}\text { Polyvinyl } \\
\text { Chloride }\end{array}$ & Silica & Acrylic Resin \\
\hline Elastic modulus & $247 \mathrm{Mpa}$ & $73 \mathrm{Gpa}$ & $1.08 \mathrm{Gpa}$ \\
Poisson ratio & 0.4 & 0.17 & 0.35 \\
Thickness & $1 \mathrm{~mm}$ & $125 \mathrm{um}$ & $125 \mathrm{um}$ \\
\hline \hline
\end{tabular}

say, the pressure applied to polymer material is converted to the axial strain of fiber. Based on Hooke's law, the axial strain $\varepsilon_{z 1}$ of the whole packaging structure can be expressed as [29]

$$
\varepsilon_{z 1}=-p \frac{\left(A_{f} v_{f}+A_{a} v_{a}+A_{p} v_{p}\right)}{\left(A_{f} E_{f}+A_{a} E_{a}+A_{p} E_{p}\right)}
$$

Where $p$ is the pressure components along y direction. $A_{f}$, $A_{a}$ and $A_{p}$ are respectively the cross-sectional area of silica fiber, acrylic resin and PVC. $v_{f}, v_{a}$ and $v_{p}$ are the Poisson ratio of silica fiber, acrylic resin and PVC, respectively. And $E_{f}, E_{a}$, and $E_{p}$ are the elastic modulus of silica fiber, acrylic resin and PVC. Similarly, the axial strain $\varepsilon_{z 2}$ of the acrylic resin coating fiber without packaging can be express as [25]

$$
\varepsilon_{z 2}=-p \frac{\left(A_{f} v_{f}+A_{a} v_{a}\right)}{\left(A_{f} E_{f}+A_{a} E_{a}\right)}
$$

According to equations (1), (3) and (9), the sensitization factor, denoted by $\mathrm{K}$, can be deduced as

$$
K=\frac{\varepsilon_{z 1}}{\varepsilon_{z 2}}=\frac{\left(A_{f} v_{f}+A_{a} v_{a}+A_{p} v_{p}\right)\left(A_{f} E_{f}+A_{a} E_{a}\right)}{\left(A_{f} E_{f}+A_{a} E_{a}+A_{p} E_{p}\right)\left(A_{f} v_{f}+A_{a} v_{a}\right)}
$$

Assuming the material parameters of $\mathrm{PVC}$, silica and acrylic resin as listed in Table I, the theoretical $\mathrm{K}$ should be 89.96 , which proves the sensitization effect of the elastic packaging.

\section{B. Vital Signs Analysis}

Respiration and heartbeat signals are regarded as the change of micro-pressure. Take a typical respiration waveform $\mathrm{R}$ and heartbeat waveform $\mathrm{H}$ of a 22-year-old male collected from the proposed mattress as an example. As plotted in Fig. 3. (a), the respiration waveform is similar to a cosine profile. It can be seen that the amplitude rises in inhaling phase and drops in expiration phase. The respiration
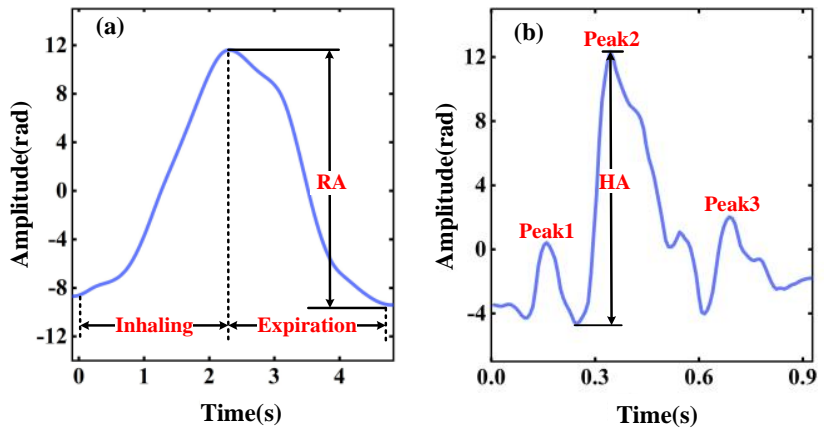

Fig. 3. The typical waveforms of (a) respiration and (b) heartbeat signal. rate can be obtained according to the repetition frequency of inhaling or expiration. Meanwhile, a typical heartbeat waveform of the male, shown in Fig. 3. (b), includes a main peak (peak2) and two lower peaks (peak1 and peak3). The main peak is caused by ventricular contraction, and the two lower peaks are triggered by atrial systole and ventricular diastole, respectively [30]. So, the heart rate can be calculated by using the repetition frequency of the main peak and the cardiovascular health can be also assessed according to the morphological characteristics of main peak [31]. In terms of recovered heartbeat and respiration waveforms, four waveform characteristics are the significant indicators to judge the physical conditions of subjects, which are heart rate (HR), respiration rate (RR), heartbeat amplitude (HA) and respiration amplitude (RA). Specifically, the four indicators can be calculated by

$$
\begin{gathered}
H R=60 \times\{\max [f f t(H)]\}_{f} \\
R R=60 \times\{\max [f f t(R)]\}_{f} \\
H A=\max (H)-\min (H) \\
R A=\max (R)-\min (R)
\end{gathered}
$$

Where, fft is Fourier transform and $\mathrm{f}$ presents the frequency of maximum in frequency spectrum. Generally, the heartbeat and respiration signals of a healthy adult at rest are continuous, with the heart rate (HR) between 60 and 100 beats per minute (bpm) [32] and the respiratory rate (RR) from 12 to 18 breaths per minute (bpm) [33].

Besides, the activity state can be judged based the frequency spectrum energy of vital signs signals. Specifically, the frequency spectrum energy, denoted by $F S E$, can be calculated by
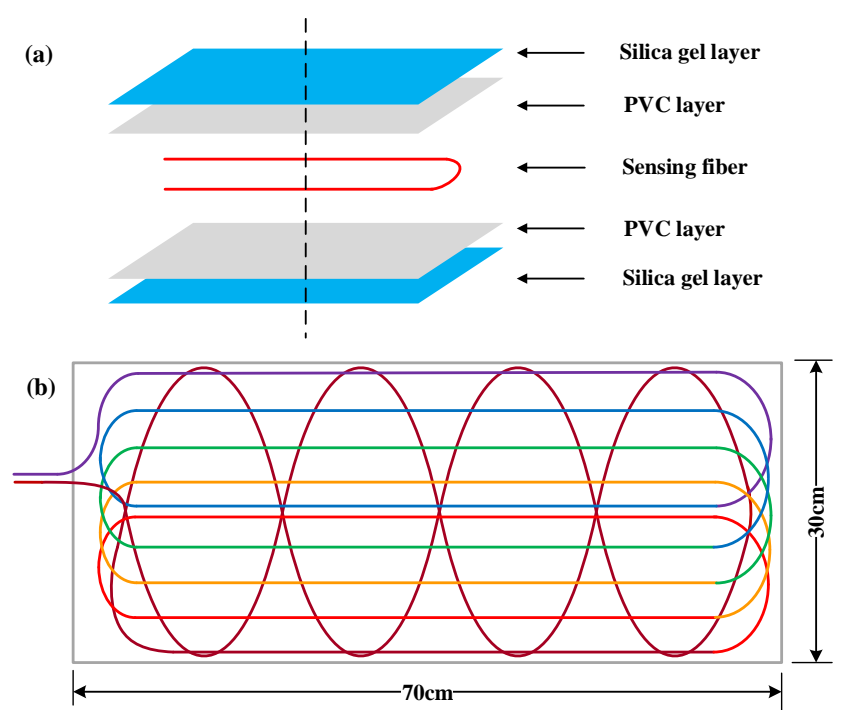

Fig. 4. (a) The structural design of the mattress; (b) The layout of optical fiber in the mattress. 


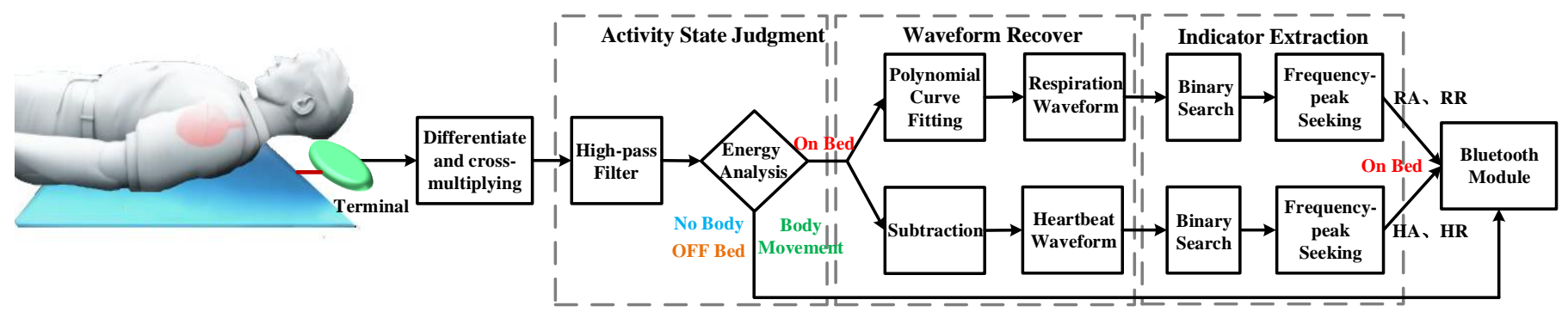

Fig. 5. System design of proposed mattress based OF-MZI

$$
F S E=\int_{0}^{2 H z} f f t(M) d f
$$

Where $\mathrm{M}$ is the mixed signals of heartbeat and respiration.

\section{System Design and Fabrication}

The proposed mattress system is composed of a sensing mattress and a terminal box. The fabrication process of sensing mattress is as follow. As the sensing fiber, a $12 \mathrm{~m}$ long single-mode fiber was deployed back-and-forth cross distribution along the $70 * 30 \mathrm{~cm}$ size PVC material, as illustrated in Fig.4. (a). Through the arrangement of fiber, the sensing blind area was eliminated and the response uniformity as well as consistency throughout the sensing mattress are enhanced. After that, another PVC material layer was tightly placed on the arranged fiber and then a preliminary packaging structure was achieved. Finally, two soft silica gel layers were respectively glued to cover the two PVC material layers for protecting the internal sensing fiber, as. shown in Fig. 4. (b).

The fiber optic mattress system was developed to continuously monitor heartbeat and respiration signals and further analyzing the vital signs. As shown in Fig. 5, the terminal box includes the light source, couplers, reference
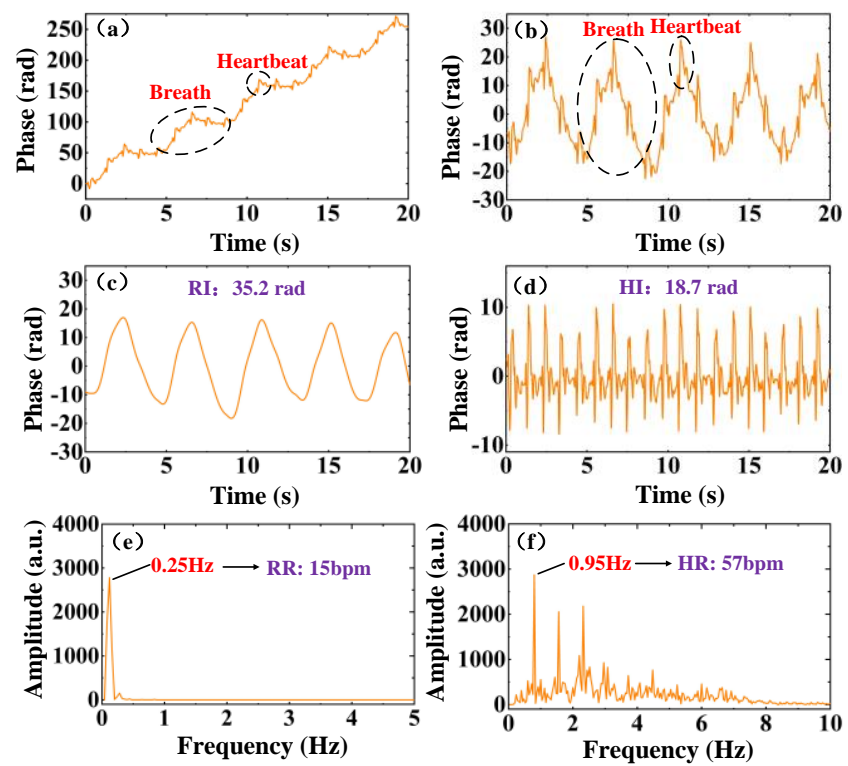

Fig. 6. (a) The raw phase signal; (b) The mixed signal of heartbeat and respiration; (c) The respiration waveform; (d) The heartbeat waveform; (e) The frequency domain of breath; (f) The frequency domain of heartbeat fiber and optical demodulator, feature extraction algorithm module and Bluetooth module. Specifically, the light source is an $1 \mathrm{~mW}$ distributed feedback laser with the central wavelength of $1550 \mathrm{~nm}$ and $3 \mathrm{~dB}$ bandwidth of $2.5 \mathrm{~Gb} / \mathrm{s}$. The sample rate of optical demodulator is $2 \mathrm{kHz} / \mathrm{s}$. According to the function, the feature extraction algorithm is roughly divided into three sections of behavior judgment, waveform recover and indicator extraction. The concrete flow of whole algorithm is presented in Fig. 5. Firstly, the raw phase signal is demodulated from the sampled signals by adopting differentiate and cross-multiplying algorithm, as depicted in Fig.6. (a). Then, high-pass filter is utilized to eliminate direct current component of raw phase signal and the mixed signal of heartbeat and respiration is obtained, as plotted in Fig.6. (b). The activity states of can be distinguished by analyzing the frequency spectrum energy of the mixed signal. If the activity state is judged as on bed, the following algorithm is carried out. Otherwise, the whole algorithm stops and the judged other activity state is immediately transmitted to the mobile by Bluetooth communication. In the following algorithm, respiration waveform can be isolated from mixed signal by employing polynomial curve fitting and the heartbeat waveform with high repetition frequency is extracted through the subtraction between mixed signal and the low-frequency breathing envelop. Figs.6. (c)-(d) illustrate the extracted respiration and heartbeat waveforms, respectively. Obviously, the low-frequency noise can be observed from the heartbeat waveform, which may result from the acoustic signals from internal organs. Afterwards, HA and RA are respectively obtained according to the peakto-peak values of heartbeat and respiration waveforms, which are searched through binary method. After that, HR and RR are calculated by seeking highest peaks in frequency domains of respiration and heartbeat waveforms, respectively, as demonstrated in Figs.6. (e)-(f). Finally, the judged activity state of on bed and four indicators of vital signs are transmitted to mobile.

Based on the above techniques, the prototype of the fiberoptic smart mattress system is developed, which is composed of a processing terminal and a mattress as displayed in Fig. 7. (a). To further evaluate the clinical performance of the fiber-optic smart mattress system, the testing sample at rest is lying on the sensing mattress and keep the shoulder on the mattress to make sure that the heart is placed within the measurement area, as is illustrated in Fig. 7. (b). 


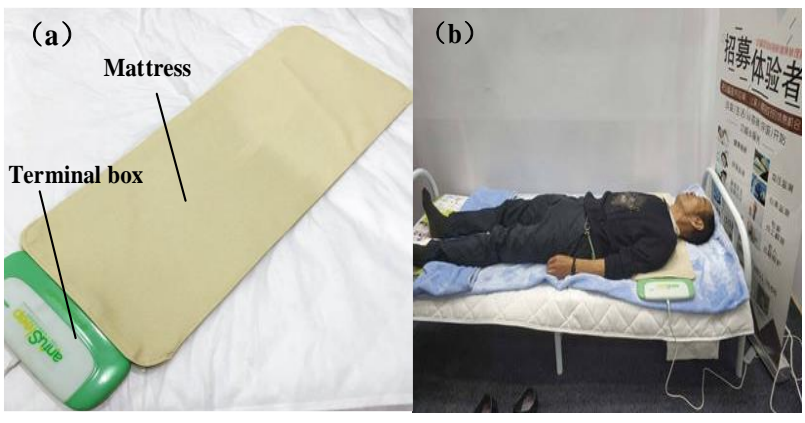

Fig. 7. (a) The prototype of fiber mattress; (b) test method.

\section{EXPERIMENT AND RESULTS}

\section{A. Subjects}

The clinical experiment involved eighteen subjects include twelve males and six females weighted between 45 $100 \mathrm{~kg}$. (All subjects voluntarily confirm their willingness to participate in the clinical test, after having been informed of all aspects of the trial that are relevant to the subject's decision to participate.) Furthermore, the measured HR from commercial oximeter (YX303) was used as reference and the reference RR was monitored by direct observation of breath counts.

\section{B. Activity Monitoring}

In the preliminary experiment, four activity states were monitored and distinguished, which include no body, on bed, body movement and off bed. Subject \#1 was monitored continuously for 25 mins under the four activity states, and the frequency spectrum energy of the mixed signal of heartbeat and respiration is depicted in Fig. 8. When nobody lay on the mattress, the frequency spectrum energy of mixed signal stayed at a low level during 5 mins. Slight fluctuations of frequency spectrum energy may be ascribed to the surrounding disturbance. After the subject lay on the mattress, the frequency spectrum energy jumped to be a higher level and keeps stabilization, which is approximate two orders of magnitude higher than that of no body. In the phase of body movement, the frequency energy further rose an order of magnitude compared with that of on bed. Besides, the frequency spectrum energy existed big fluctuation, which is caused by drastic change of body movement. When the subject left the bed, the corresponding frequency spectrum energy immediately jumped to a lower level but was still higher than that of No Body at first, and then gradually dropped to the same level as no body. In the whole process, it takes about 10 mins to be stabilized, owing to the elastic aftereffect of soft silica gel material. Apparently, there is clear distinction between the frequency energy scopes of the four activity states. Therefore, the proposed fiber optic mattress is capable of easily distinguishing different activity condition.

\section{Heartbeat and respiration indicators Analysis}

For testing the ability of mattress prototype to diagnose the abnormal health conditions of subjects, four waveform characteristics corresponding to heartbeat and respiration indicators of five typical subjects were analyzed. Specifically, subjects \#4 and \#6 have the symptom of bradycardia and subject \#10 has the symptom of tachycardia. Subject \#13 and subject \#17 suffer from apnoea and polypnea, respectively. Fig. 9 illustrates the measurement results of the five subjects. As shown in Figs. 9 (a) and (b), although the HA of subject \#4 and subject \#6 are steady, their HR values are significantly lower than the normal range and diagnosed as bradycardia. Besides, the subject $\# 10$ is defined as tachycardia on account of higher HA and HR beyond normal range. It should be mentioned that bradycardia and tachycardia are the most common symptoms of arrhythmia, which may result in fatigue, chest distress, dizziness, even fainting and sudden death [34], and is also relevant to the cardiac diseases of myocarditis, coronary disease, valvular heart disease and congenital heart disease. Therefore, the mattress system shows potential in the detection of arrhythmia, which is significant in early diagnosis and prevention of relevant cardiac diseases.

In addition, the subject $\# 13$ and subject $\# 17$ have the abnormal respiration states. As demonstrated in Fig. 9. (d), the RR and RA of subject \#13 are all continuously declining from the third minute. Finally, the RR drops to $0 \mathrm{bpm}$ and the RA becomes very weak with slight fluctuation, which may be induced by internal organs. According to above

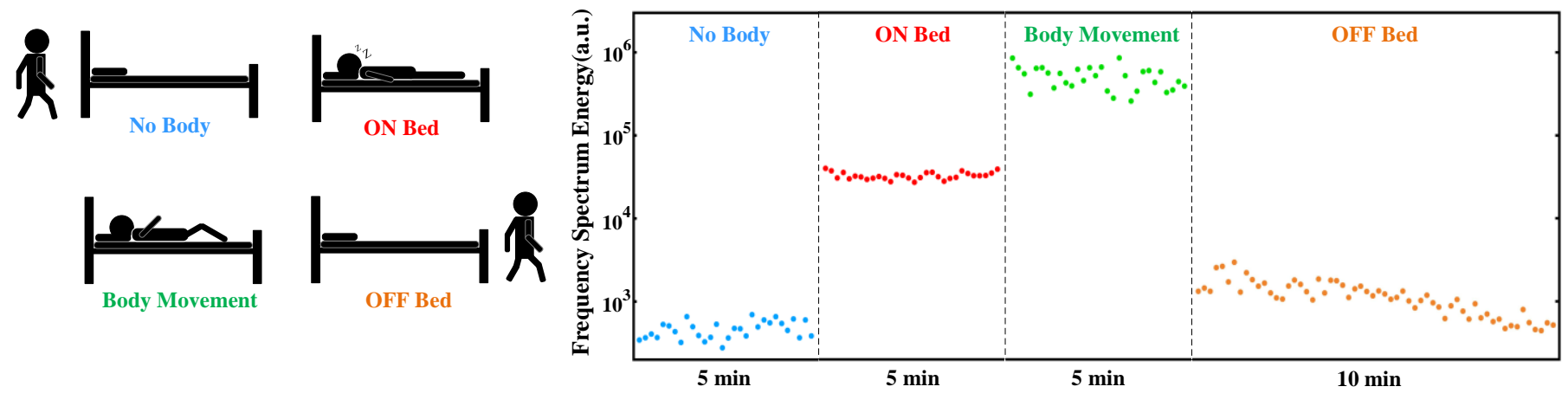

Fig. 8. Frequency spectrum energy analysis of four activity states 


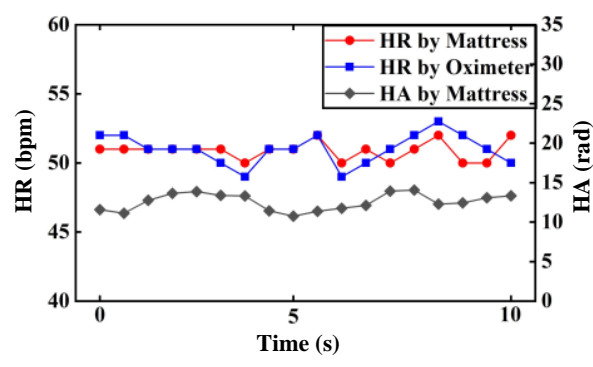

(a) Subject \#4

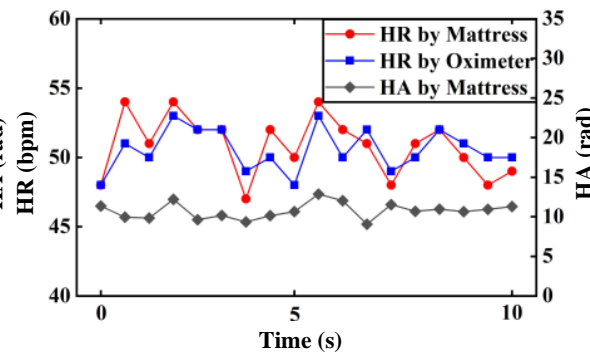

(b) Subject \#6

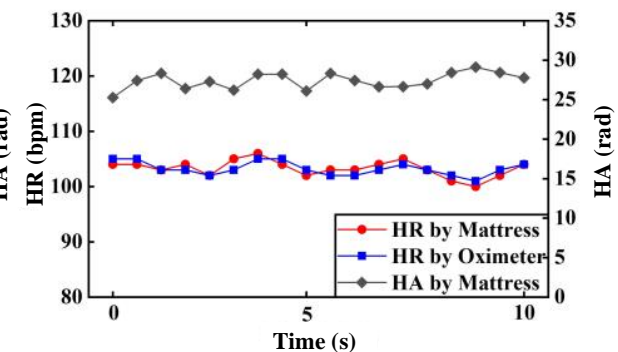

(c) Subject \#10

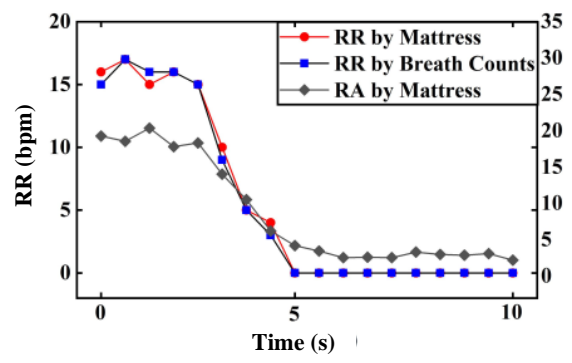

(d) Subject \#13

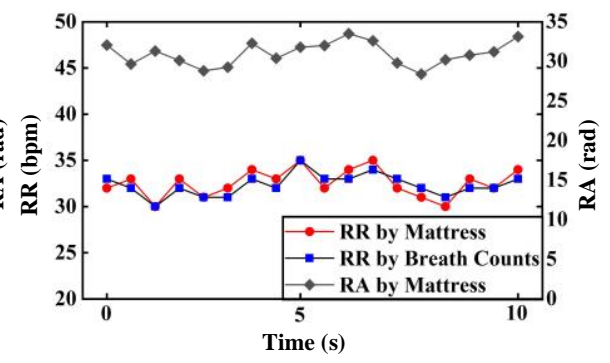

(e) Subject \#17

Fig. 9. Heartbeat and respiration indicators measurement results

respiratory phenomenon, the symptom of subject \#13 is diagnosed as apnoea. From Fig. 9. (e), it can be seen that the $\mathrm{RR}$ of subject \#17 is from 30 to $35 \mathrm{bpm}$, which is far above normal RR. Besides, the RA has a higher amplitude. So, it can be inferred that subject $F$ has the symptom of polypnea. Notably, the symptoms of polypnea and apnoea are relatively common in abnormal breathing, which are usually associated with respiratory diseases, such as asthma, emphysema, chronic obstructive pulmonary and so on. Thus, the mattress system is also promising in the early detection and diagnosis of the respiratory diseases.

\section{Error and Accuracy Analysis}

The total experiment results of eighteen subjects are summarized in Table II, which includes the mean and standard deviation (SD) of the four indicators. It can be seen that the calculated values of HR and RR are mostly close to the corresponding reference values of HR and RR. The absolute errors of HR and RR are less than $2 \mathrm{bpm}$ and $1 \mathrm{bpm}$. The largest SD of calculated HR and RR of all the subjects are $3.0 \mathrm{bpm}$ and $2.6 \mathrm{bpm}$, which is acceptable according to the American National Standard ANSI/AAMI EC13: 2002 [35].

Furthermore, we chose sample data from the eighteen subjects to analyze the determination capability of the mattress system in monitoring HR and RR. Figs. 10 (a)-(b) show the Bland-Altman plots for the performance of the mattress system and the reference. The mean difference (MD) between calculated HR and reference HR is $0.11 \mathrm{bpm}$ and the MD of them is $0.11 \mathrm{bpm}$. Besides, the standard deviation

TABLE II

CALCULATED RESULTS MEASURED BY FIBER OPTIC MATTRESS AND THE REFERENCE RESULTS MEASURED BY COMMERCIAL DEVICES

\begin{tabular}{|c|c|c|c|c|c|c|c|c|}
\hline \multirow{2}{*}{$\frac{\text { Subject }}{\text { No. }}$} & \multirow[t]{2}{*}{ Gender } & \multirow[t]{2}{*}{ Weight } & \multicolumn{2}{|c|}{ Mean HR \pm SD } & \multicolumn{2}{|c|}{ Mean $\mathrm{RR} \pm \mathrm{SD}$} & \multirow{2}{*}{$\frac{\mathrm{HA}}{\mathrm{Cal} .}$} & \multirow{2}{*}{$\frac{\mathrm{RA}}{\mathrm{Cal} .}$} \\
\hline & & & Ref. & Cal. & Ref. & Cal. & & \\
\hline \#1 & Male & 56 & $72.8 \pm 1.4$ & $73.0 \pm 1.5$ & $16.6 \pm 0.8$ & $16.5 \pm 1.6$ & $15.5 \pm 1.4$ & $40.1 \pm 4.4$ \\
\hline \#2 & Female & 45 & $69.3 \pm 2.2$ & $70.2 \pm 1.2$ & $15.3 \pm 1.0$ & $15.6 \pm 1.2$ & $5.8 \pm 0.7$ & $21.3 \pm 1.3$ \\
\hline \#3 & Female & 55 & $81.8 \pm 1.8$ & $82.0 \pm 3.0$ & $14.2 \pm 1.2$ & $14.3 \pm 1.2$ & $11.7 \pm 0.8$ & $30.4 \pm 4.1$ \\
\hline$\# 4$ & Male & 67 & $51.1 \pm 2.3$ & $51.0 \pm 0.0$ & $11.0 \pm 0.6$ & $11.3 \pm 1.0$ & $12.7 \pm 1.1$ & $23.8 \pm 1.5$ \\
\hline$\# 5$ & Male & 57.5 & $60.0 \pm 2.2$ & $60.6 \pm 2.5$ & $15.4 \pm 0.5$ & $15.0 \pm 0.0$ & $12.6 \pm 2.8$ & $20.8 \pm 2.6$ \\
\hline \#6 & Male & 62.5 & $51.0 \pm 2.3$ & $51.8 \pm 2.2$ & $15.2 \pm 1.7$ & $15.3 \pm 2.0$ & $10.5 \pm 1.0$ & $21.4 \pm 2.9$ \\
\hline \#7 & Female & 47 & $66.2 \pm 2.4$ & $66.5 \pm 2.9$ & $19.0 \pm 0.8$ & $19.0 \pm 1.5$ & $8.4 \pm 0 . .6$ & $37.9 \pm 1.1$ \\
\hline$\# 8$ & Male & 70 & $59.6 \pm 2.3$ & $59.5 \pm 2.2$ & $23.2 \pm 1.7$ & $23.3 \pm 1.6$ & $12.5 \pm 1.3$ & $17.0 \pm 1.1$ \\
\hline \#9 & Male & 100 & $59.6 \pm 1.8$ & $59.0 \pm 2.0$ & $19.6 \pm 0.8$ & $19.5 \pm 1.6$ & $11.7 \pm 1.2$ & $29.2 \pm 2.3$ \\
\hline$\# 10$ & Male & 59 & $104.0 \pm 0.9$ & $103.6 \pm 1.0$ & $14.6 \pm 0.8$ & $14.6 \pm 1.0$ & $27.1 \pm 1.5$ & $24.4 \pm 1.7$ \\
\hline \#11 & Male & 72 & $63.6 \pm 3.2$ & $63.5 \pm 3.0$ & $20.5 \pm 0.8$ & $20.5 \pm 0.8$ & $14.1 \pm 1.2$ & $18.1 \pm 2.4$ \\
\hline \#12 & Female & 48 & $63.5 \pm 3.2$ & $70.0 \pm 1.5$ & $21.5 \pm 1.0$ & $21.3 \pm 1.3$ & $11.1 \pm 0.6$ & $14.1 \pm 1.8$ \\
\hline$\# 13$ & Male & 55 & $81.1 \pm 1.2$ & $80.5 \pm 0.8$ & $0.0 \pm 0.0$ & $0.0 \pm 0.0$ & $20.0 \pm 1.5$ & $1.88 \pm 0.5$ \\
\hline$\# 14$ & Male & 64 & $61.4 \pm 2.5$ & $61.2 \pm 2.6$ & $22.6 \pm 1.1$ & $22.6 \pm 1.5$ & $15.1 \pm 1.0$ & $23.5 \pm 1.4$ \\
\hline$\# 15$ & Female & 60 & $58.0 \pm 2.2$ & $58.8 \pm 2.3$ & $16.6 \pm 3.1$ & $16.8 \pm 2.6$ & $13.2 \pm 0.7$ & $26.7 \pm 2.4$ \\
\hline \#16 & Male & 61 & $67.8 \pm 1.2$ & $67.8 \pm 1.2$ & $17.6 \pm 1.3$ & $17.0 \pm 1.5$ & $10.6 \pm 1.5$ & $21.9 \pm 2.7$ \\
\hline$\# 17$ & Female & 53 & $74.6 \pm 1.8$ & $74.1 \pm 1.7$ & $32.3 \pm 1.0$ & $31.8 \pm 1.1$ & $21.1 \pm 1.4$ & $30.2 \pm 1.2$ \\
\hline$\# 18$ & Male & 70 & $65.6 \pm 1.8$ & $65.0 \pm 1.4$ & $17.8 \pm 1.3$ & $18.0 \pm 1.8$ & $15.4 \pm 0.7$ & $24.8 \pm 2.0$ \\
\hline
\end{tabular}

Ref. denotes the measured reference results and Cal. denotes the results monitored by our system. The unit of weight is Kg. The units of HR and RR are bpm and the units of HA and RA are rad. 

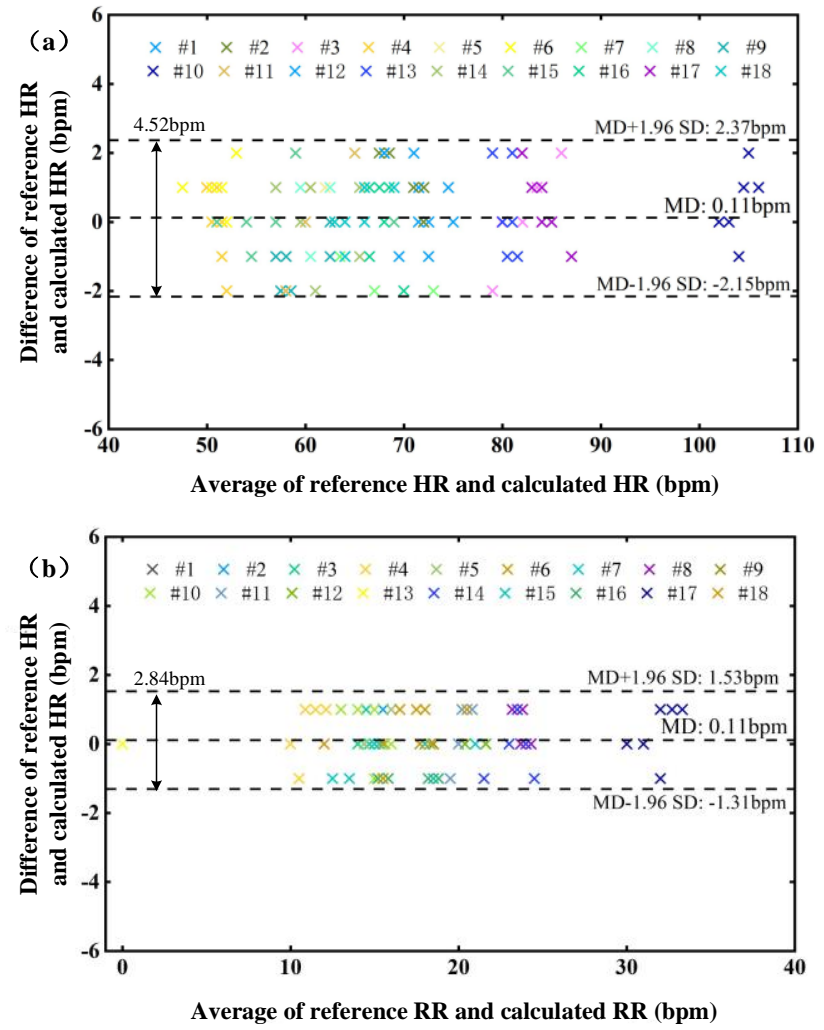

Fig. 10. Bland-Altman plots for the performance of the mattress system and the reference

(SD) of the difference between calculated HR and reference $\mathrm{HR}$ is $1.15 \mathrm{bpm}$ and the SD of the difference between them is $0.72 \mathrm{bpm}$. Based on Bland-Altman analysis method [36], the limits of agreement (LOA) of HR and RR are from -2.15 $\mathrm{bpm}$ to $2.37 \mathrm{bpm}$, and from $-1.31 \mathrm{bpm}$ to $1.53 \mathrm{bpm}$, respectively. Apparently, all values lie within the LOA range. So, it can be inferred that the reproducibility of fiber optic mattress is good [37]. The results suggest that the excellent reproducibility and accuracy of the proposed mattress system.

\section{E. Reliability Test}

In order to test the reliability of the fiber optic mattress, two experiments were carried out, including reliability texting of long-term continuous monitoring and the response consistency testing at different positions of the mattress.

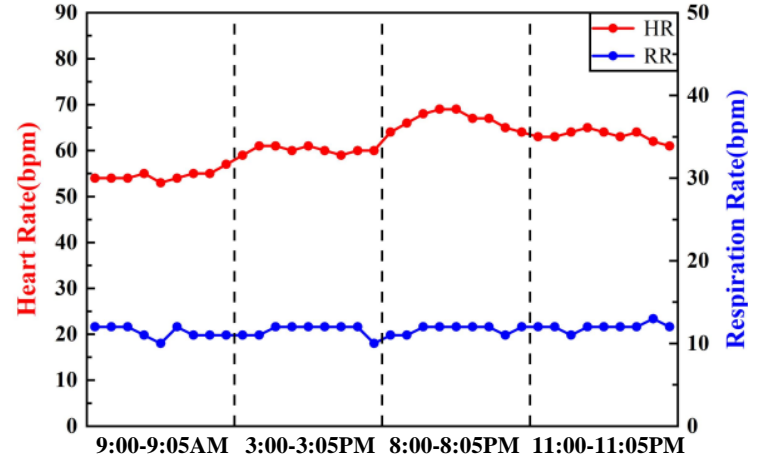

Fig. 11. HR and RR monitoring of subject \#9 from morning to night.

Primarily, the subject \#9 was continuously monitored in the four periods of morning, afternoon, evening and night, of which the results were depicted in Fig. 11. Obviously, the $\mathrm{RR}$ of the subject stays roughly stable and is within the normal RR scope. Whereas, measured HR values are slightly different at the four periods. Specifically, the HR values monitored in the morning are lower, while in the evening they are higher. On the whole, HR presents the trend of rising from morning to evening and shows the downtrend from evening to night, which is consistent with daily change of HR. As a consequence, the fiber optic mattress system performs well in the long-time monitoring.

In the following experiment, we tested the response consistency at different positions of mattress. Lying at the six different positions of fiber optic mattress as illustrated in Fig. 12 , subject \#15 was respectively monitored for $5 \mathrm{~min}$. It can be seen that $\mathrm{HR}$ and $\mathrm{RR}$ values measured at different positions are steady with slight fluctuations. Further, Table III shows the statistic measurement results at different positions, which include mean, standard deviation (SD), difference of HR and difference of RR. It is clear that the mean and SD of HR and RR values at different positions are very close, and the differences of $\mathrm{HR}$ and $\mathrm{RR}$ are all less than $5 \%$, which can be considered as the normal fluctuation of HR and RR. Hence, the fiber optic mattress has better robustness in clinic application.

TABLE III

STATISTIC RESULTS OF HR AND RR MEASURED AT DIFFERENT LOCATIONS

\begin{tabular}{ccccccc}
\hline Position & 1 & 2 & 3 & 4 & 5 & 6 \\
\hline $\begin{array}{c}\text { Rean HR } \pm \text { SD } \\
(\mathrm{bpm})\end{array}$ & $56.4 \pm 0.5$ & $58.3 \pm 1.0$ & $58.3 \pm 1.3$ & $56.7 \pm 0.9$ & $57.4 \pm 1.2$ & $58.2 \pm 1.1$ \\
$\begin{array}{c}\text { Mean RR } \pm \text { SD } \\
(\mathrm{bpm})\end{array}$ & $17.4 \pm 0.8$ & $17.6 \pm 1.0$ & $16.6 \pm 0.8$ & $17.2 \pm 1.1$ & $16.7 \pm 1.1$ & $16.8 \pm 0.6$ \\
$\begin{array}{c}\text { Difference of HR } \\
\text { Difference of RR }\end{array}$ & 0 & $3.4 \%$ & $3.4 \%$ & $0.5 \%$ & $1.8 \%$ & $3.2 \%$ \\
\hline
\end{tabular}

Difference of HR denotes the error between the HR measured at position 1 and the HR measured at other positions. Difference of RR denotes the error between the RR measured at position 1 and the RR measured at other positions. 


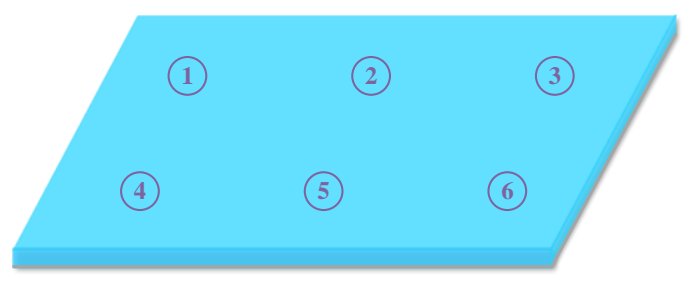

\section{CONCLUSION}

We have proposed and demonstrated a smart mattress prototype system based on optical fiber Mach-zender interferometer (OFMZI) for monitoring heartbeat and respiration simultaneously. Specifically, the sensing fiber of OFMZI is placed in the mattress and the reference fiber is arranged in the terminal. Beneficial from the phase modulation mechanism and package sensitization, high sensitivity to micro-pressure is realized. By employing feature extraction algorithm, four activity states can be distinguished and four indicators of vital signs can be extracted. In the clinical experiment, eighteen subjects were tested and five of them are diagnosed as the symptoms of abnormal heart rate and respiration. The error analysis shows that the measurement errors of the heart rate and respiration rate were less than $2 \mathrm{bpm}$ and $1 \mathrm{bpm}$, respectively. In addition, the test of long-time monitoring and consistency at different positions confirms the reliability of the fiber optic mattress. The smart mattress system provides a convenient way for reliable household healthcare monitoring and hospital health monitoring due to the advantages of low cost, soft, simple fabrication and high comfortability.

\section{REFERENCES}

[1] M. C. Yu, H. Wu, J. L. Liou, M. S. Lee, and Y. P. Hung, "Multiparameter sleep monitoring using a depth camera," in Proc. 5th Int. Joint. Conf. Biomed. Eng. Syst. Technol., 2012, pp. 311-325.

[2] S. D. Min et al., "Noncontact respiration rate measurement system using an ultrasonic proximity sensor," IEEE Sensors. J., vol. 10, no. 11, pp. 1732-1739, Nov. 2010.

[3] X. Chen et al., "A breath sensor using carbon nanotubes operated by field effects of polarization and ionization," Sensors \& Actuators A: Physical, vol. 158, no. 2, pp. 328-334, Mar. 2010.

[4] H. J. Baek, G. S. Chung, K. K. Kim, and K. S. Park, "A smart health monitoring chair for nonintrusive measurement of biological signals," IEEE Trans. Inf. Technol. Biomed., vol. 16, no. 1, pp. 150-158, Jan. 2012.

[5] F. Wang, M. Tanaka, and S. Chonan, "Development of a PVDF piezopolymer sensor for unconstrained in-sleep cardiorespiratory monitoring," J. Intel. Mat Syst. Str., vol. 14, no. 3, pp. 185-190, Mar. 2003.

[6] J. Alihanka, K. Vaahtoranta, and I. Saarikivi, "A new method for longterm monitoring of the ballistocardiogram, heart rate, and respiration," Am. J. Physiol., vol. 240, no. 5, pp. 348-392, May. 1981.
[7] O. T. Inan, D. Park, L. Giovangrandi, and G. Kovacs, "Noninvasive measurement of physiological signals on a modified home bathroom scale," IEEE Trans. Biomed. Eng., vol. 59, no. 8, pp. 2137-2143, Aug. 2012.

[8] G. Matthews, B. Sudduth, and M. Burrow, "A non-contact vital signs monitor," Crit. Rev. Biomed. Eng., vol. 28, no. 1/2, pp. 173-178, 2000.

[9] M. V. Scanlon, "Acoustically monitor physiology during sleep and activity," in Proc. 1st Joint BMES/EMBS Conf., 21st Annu. Conf. IEEE Eng. Med. Biol. Annu. Fall Meeting Biomed. Eng. Soc., vol. 2, p. 787, 1999.

[10] Y. Chee, J. Han, J. Youn, and K. Park, "Air mattress sensor system with balancing tube for unconstrained measurement of respiration and heart beat movements," Physiol. Meas., vol. 26, no. 4, pp. 413-422, 2005.

[11] Y. Mengsun, J. Yang, and Y. Zhou, "Study on sleep monitering with micromovement sensitive mattress monitering system," Chin. J. Aerospace. Med., vol. 10, no. 1, pp. 40-45, May. 1999.

[12] J. Wo et al., "Noninvasive respiration movement sensor based on distributed Bragg reflector fiber laser with beat frequency interrogation," J. Biomed. Opt., vol. 19, no. 1, 017003, Jan. 2014.

[13] L. Dziuda, F. W. Skibniewski, M. Krej, and J. Lewandowski, "Monitoring respiration and cardiac activity using fiber Bragg gratingbased sensor," IEEE Trans. Biomed. Eng., vol. 59, no. 7, pp. 19341942, Jul. 2012.

[14] L.-G. Lindberg, H. Ugnell, and P. Å. Öberg, "Monitoring of respiratory and heart rates using a fibre-optic sensor," Med. Biol. Eng. Comput., vol. 30, no. 5, pp. 533-537, 1992.

[15] A. G. Leal-Junior et al., "Polymer optical fiber-based sensor for simultaneous measurement of breath and heart rate under dynamic movements," Optics and Laser Technol., vol. 109, pp. 429-436, 2019.

[16] M. Nishiyama, M. Miyamoto, and K. Watanabe, "Respiration rhythm monitoring in sleep based on weight movement using hetero-core fiber optic sensors," in Proc. 2010 IEEE Int. Conf. Control, Automation and Systems, pp. 205-208.

[17] F. C. Fávero, V. Pruneri, and J. Villatoro, "Microstructured optical fiber interferometric breathing sensor," J. Biomed. Opt., vol. 17, no. 3 , 037006, Mar. 2012.

[18] J. Mathew, Y. Semenova, and G. Farrell, "A miniature optical breathing sensor," Biomed. Opt. Express, vol. 3, no. 12, pp.3325-3331, 2012.

[19] Y. Zhang, Z. Chen, and H. I. Hee, "Noninvasive Measurement of Heart Rate and Respiratory Rate for Perioperative Infants," $J$. Lightwave. Technol., vol. 37, no. 11, pp. 2807-2814, 2019.

[20] R. Gao, D. F. Lu, M. Zhang, and Z. M. Qi, "Vibration sensor based on the resonance power leakage in a tapered capillary fiber," IEEE Sensors J., vol. 17, no. 24, pp. 8332 - 8337, Dec. 2017.

[21] M. D. Todd, M. Seaver, and F. Bucholtz, "Improved, operationallypassive interferometric demodulation method using 3/spl times/3 coupler," Electronics Lett., vol. 38, no. 15, pp. 784-786, Jul. 2002.

[22] A Masoudi, M. Belal, and T. P. Newson, "A distributed optical fibre dynamic strain sensor based on phase-OTDR," Meas. Sci. Technol., vol. 24 , no. 8, 085204, Jul. 2013. 
[23] C. Wang et al., "Distributed OTDR-interferometric sensing network with identical ultra-weak fiber Bragg gratings," Opt. Express, vol. 23, no. 22, pp. 29038-29046, Oct 2015.

[24] C. Wang, C. Wang, Y. Shang, X. Liu, and G. Peng, "Distributed acoustic mapping based on interferometry of phase optical timedomain reflectometry," Opt. Commun., vol. 346, pp. 172-177, Jul. 2015.

[25] W. Zhang et al., "Microstructured Optical Fiber Based Distributed Sensor for In Vivo Pressure Detection," J. Lightwave. Technol., vol. 37, no. 9, pp. 1865-1872, May. 2019.

[26] S. Ziraki, S. M. Zebarjad, and M. J. Hadianfard, "A study on the tensile properties of silicone rubber/polypropylene fibers/silica hybrid nanocomposites," J. Biomed. Mater. Res. A, vol. 57, pp. 289-296, 2016.

[27] J. T.-M. Cheung, M. Zhang, A. K. Leung, and Y. B. Fan, "Threedimensional finite element analysis of the foot during standing-a material sensitivity study," J. Biomech., vol. 38, no. 5, pp. 1045-1054, 2005.

[28] S. C. Tjin, R. Suresh, and N. Q. Ngo, "Fiber Bragg grating based shearforce during standing - a material sensitivity study," J. Biomech., vol. 38, no. 5, pp. 1045-1054, 2005.

[29] G. L. Shen, Mechanics of Composite Materials. 2nd ed., Beijing, CN: Tsinghua University Press, 2013, pp. 214-221.

[30] F. S. Almehmadi, and M. R. Chatterjee, "Secure chaotic transmission of electrocardiography signals with acousto-optic modulation under profiled beam propagation," Appl. Optics, vol. 54, no. 2, pp. 195-203, Jan. 2015.

[31] G. E. McVeigh et al., "Age-related abnormalities in arterial compliance identified by pressure pulse contour analysis: aging and arterial compliance," Hypertension, vol. 33, no. 6, pp. 1392-1398, 1999.

[32] All About Heart Rate (Pulse). American Heart Association. 22 Aug. 2017. [Online]. Available: http://wikipedia.moesalih.com/Heart_rate\#cite_ref-aha_rest_1-0

[33] KE Barrett et al., Ganong's review of medical physiology (24 ed.), ISBN 0071780033

[34] M. C. Sanguinetti, and M. Tristani-Firouzi, "hERG potassium channels and cardiac arrhythmia," Nature, vol. 440, pp. 463-469, Mar. 2006

[35] Cardiac Monitors, Heart Rate Meters, and Alarms, American National Standard ANSI/AAMI EC13:2002, Association for the Advancement of Medical Instrumentation, Arlington, VA, USA, 2002.

[36] J. Martin Bland, and D. G. Altman, "Statistical methods for assessing agreement between two methods of clinical measurement," The Lancet, vol. 327, no. 8476, pp. 307-310, Feb. 1986.

[37] Ł. Dziuda, M. Krej, and F. W. Skibniewski, "Fiber Bragg grating strain sensor incorporated to monitor patient vital signs during MRI," IEEE Sensors J., vol. 13, no. 12, pp. 4986-4991, Dec. 2013 